\title{
Homological optimality in Discrete Morse Theory through chain homotopies
}

\author{
Helena Molina-Abril *, Pedro Real \\ Department of Applied Mathematics, University of Seville, Spain
}

\section{A R T I C L E I N F O}

\section{Article history:}

Available online 16 February 2012

\section{Keywords:}

Discrete Morse Theory

Gradient vector field

Cell complex

Integral-chain complex

Chain homotopy

Graph

\begin{abstract}
A B S T R A C T
Morse theory is a fundamental tool for analyzing the geometry and topology of smooth manifolds. This tool was translated by Forman to discrete structures such as cell complexes, by using discrete Morse functions or equivalently gradient vector fields. Once a discrete gradient vector field has been defined on a finite cell complex, information about its homology can be directly deduced from it. In this paper we introduce the foundations of a homology-based heuristic for finding optimal discrete gradient vector fields on a general finite cell complex $K$. The method is based on a computational homological algebra representation (called homological spanning forest or HSF, for short) that is an useful framework to design fast and efficient algorithms for computing advanced algebraic-topological information (classification of cycles, cohomology algebra, homology $A(\infty)$-coalgebra, cohomology operations, homotopy groups, ...). Our approach is to consider the optimality problem as a homology computation process for a chain complex endowed with an extra chain homotopy operator.
\end{abstract}

(c) 2012 Published by Elsevier B.V.

\section{Introduction}

Morse theory (Milnor, 1963) has been considered a powerful tool in its applications to computational topology, computer vision and geometric modeling. In (Forman, 1995) the author formulates a version of this theory for discrete structures such as cell complexes. The aim of Forman's Discrete Morse Theory is to find simplicial collapses (cell pairings) that transform the initial complex to a simpler complex. This theory relies either on admissible functions on a cell complex, called discrete Morse functions, or equivalently gradient vector fields.

Forman proved that the topology of a cell complex can be partly read out of the critical cells of a discrete gradient vector field defined on it. Critical cells are cells that are not paired with any other cell in the gradient vector field. A gradient vector field is considered optimal if it has the minimum possible number of critical cells.

In (Lewiner et al., 2003) the authors develop a heuristic for computing optimal Morse pairings. This heuristic computes optimal gradient vector fields for combinatorial 2-manifolds. However, for general cell complexes this problem has not yet been solved.

The long term motivation of the work presented here is to develop a computational homological algebra framework for a finite cell complex $K$ embedded in $\mathbb{R}^{n}$, based on Discrete Morse Theory notions which gives positive and efficient answers to the problem of calculating classical algebraic topological information (Euler characteristic, Betti numbers, classification of cycles, cohomology

\footnotetext{
* Corresponding author.

E-mail address: habril@us.es (H. Molina-Abril).
}

algebra, cohomology operations, fundamental group, homology $A(\infty)$-coalgebra, homotopy groups, ...). Due to the limited space of the paper, we exclusively focus here on purely topological results. The interplay of this framework with geometric or analytical information of a geometric object and the introduction of new algebraic operators in our framework "measuring" advanced algebraic-topological invariants will be treated elsewhere.

The optimal gradient vector field notion has already shown its power for computing advanced topological invariants more complex than homology, like cohomology algebra (see Forman (2002)). In this paper we integrate the notion of discrete gradient vector field into a general computational homological algebra scheme and we create a homology-based heuristic for finding optimal discrete gradient vector fields. The underlying idea is to classify chain homotopies as gradient vector fields on a finite cell complex and vice versa. In order to do this, we use a chain homotopy operator (see González-D́raz and Real (2005), González-Díiz et al. (2009)) at algebraic level, and its associated graph-based representation (homological spanning forest or, HSF for short Molina-Abril and Real (2009)) at combinatorial level.

The paper is organized as follows: In Section 2 some basic definitions are introduced. The theoretical foundation of our work is detailed in Section 3. In Section 4 the relation between this algebraic machinery and the optimality in Discrete Morse Theory are established. We finish the paper with some examples and conclusions.

\section{Preliminaries}

In this section, some basic definitions are introduced in order to understand the proposed algebraic-topological approach. We 
present the notion of (combinatorial) cell complex in a finitedimensional Euclidean space with the cell boundary information described in algebraic terms.

\subsection{Cell complexes}

The ring of coefficients $\Lambda$ is a commutative field (for example, a finite field, the rational numbers, the real numbers, ...). Let $\left\{x_{1}, x_{2}, \ldots, x_{n}\right\}$ be a finite set of symbols. We denote by $\Lambda\left[x_{1}, \ldots, x_{n}\right]$ the module of formal linear combinations $\lambda_{1} x_{1}+\lambda_{2} x_{2}+\cdots \lambda_{n} x_{n}$, with $\lambda_{i} \in \Lambda$.

Let $\ell$ be a positive integer. Let $\mathbb{B}^{\ell}=\left\{x \in \mathbb{E}^{\ell}\right.$ s.t. $\left.|x| \leqslant 1\right\}$ be the closed unit ball in the $\ell$-dimensional Euclidean space $\mathbb{E}^{\ell}$. The boundary of $\mathbb{B}^{\ell}$ is the unit $(\ell-1)$-sphere $\mathbb{S}^{(\ell-1)}$ and the interior of $\mathbb{B}^{\ell}$, denoted by $\operatorname{Int} \mathbb{B}^{\ell}$, is the open unit $\ell$-dimensional ball Int $\mathbb{B}^{\ell}=\left\{x \in \mathbb{E}^{\ell}\right.$ s.t. $\left.|x|<1\right\}$. A $p$-cell in $\mathbb{E}^{\ell}$ (with $0<p \leqslant \ell$ ) is a subset of $\mathbb{E}^{\ell}$ which is homeomorphic (same topology) to the open unit ball Int $\mathbb{B}^{p}$ (that is, to $\mathbb{R}^{p}$ ).

A 0-cell is a topological space homeomorphic to a point of a finite-dimensional Euclidean space. The dimension of a $p$-cell $\sigma$ is $|\sigma|=p$ and the notation $\sigma^{(p)}$ indicates that $\sigma$ is a cell of dimension $p$. The union as point set of a set of cells $K$ in $\mathbb{E}^{\ell}$ is called the carrier of $K$ and it is denoted by $|K|$. Let us define the boundary of a $p$-cell $\sigma$ as $\partial \sigma=\bar{\sigma} \backslash \sigma$, where $\bar{\sigma}$ is the closure of $\sigma$. To indicate relationships between cells, we write $\tau>\sigma$ (or $\sigma<\tau$ ) and we say that $\sigma$ is a face of $\tau$ if $\sigma \neq \tau$ and $\sigma \subset \bar{\tau}$, where $\bar{\tau}$ is the closure of $\tau$. We write $\tau \geqslant \sigma$ if either $\tau=\sigma$ or $\tau>\sigma$.

A cell complex $K=\left\{K_{i}\right\}_{i=0}^{\ell}$ embedded in $\mathbb{E}^{\ell}$ is a finite collection of $n$ cells $\left\{\sigma_{i}^{(r)} \in K_{r}\right\}_{i=1}^{n}$ of different dimensions $0 \leqslant r \leqslant \ell$ such that:

(i) $|K|=\bigcup_{i=1}^{n} \sigma_{i}=\left|K_{0}\right| \cup\left|K_{1}\right| \cup \ldots \cup\left|K_{\ell}\right|$,

(ii) $\sigma_{i} \cap \sigma_{j}=\emptyset(i \neq j)$,

(iii) if $\operatorname{dim}\left(\sigma_{i}\right)=p$, then $\partial \sigma_{i} \subset \bigcup_{i=1}^{p-1} K_{i}$ (where $0 \leqslant p \leqslant \ell$ ).

The set $K_{r}$ consists of all the $r$-cells of $K$, for $0 \leqslant r \leqslant \ell$. It is possible that $K_{i}=\emptyset$ for some $0<i \leqslant \ell$.

We say that a cell $\sigma$ is a facet of a cell $\tau$ when $\sigma$ is a proper face of $\tau$ of maximal dimension.

If all the cells of $K$ are convex sets of $\mathbb{E}^{\ell}$, then $K$ is called convex cell complex. Simplicial, cubical and some polyhedral complexes are special cases of convex cell complexes. A cell complex is specified by the face poset (the partial order determined by the cells) and its boundary relations.

\subsection{Homology of chain complexes}

Roughly speaking, the idea of homology of a cell complex consists of analyzing its degree of connectivity by using formal sums of cells.

A differential operator for a cell complex $K$ with coefficients in $\Lambda$ is a linear map $d: \Lambda[K] \rightarrow \Lambda[K]$, such that the image of a $p$-cell $\sigma$ is a linear combination of some $(p-1)$-cells of the boundary $\partial(\sigma)$ and satisfying $d \circ d=0$. Considering a cell complex $K$ embedded in $\mathbb{E}^{\ell}$, its geometric realization $|K|$ is a regular triangulable cell complex and therefore a differential operator $\partial$ with coefficients in the field $\Lambda$ can always be defined. This operator, called boundary operator, completely determines the singular homology of $|K|$ (Hatcher, 2001) (see Fig. 1).

The chain complex canonically associated to the cell complex $K$ is the graded differential vector space $\left(C_{*}(K), \partial\right)$, where $C_{p}(K)=\Lambda\left[K_{p}\right]$, for all $p=0,1, \ldots r$, and $\partial: C_{*}(K) \rightarrow C_{*-1}(K)$ is the previous boundary operator for the cell complex $K$. For instance, to find a boundary operator $\partial$ for a simplicial complex is straightforward, but it is not, in general, an easy task for others cell complexes. The following is one of the fundamental results in the theory of cell complexes (see for instance (Hatcher, 2001)).

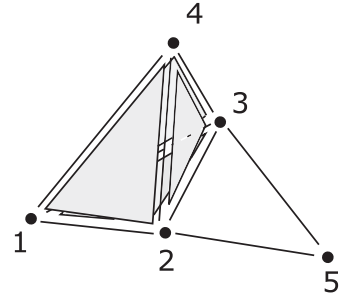

Fig. 1. A cell complex.

Theorem 1. Let $K$ a finite cell complex. There are algebraic boundary maps $\partial_{p}: C_{p}(K, \Lambda) \rightarrow C_{p-1}(K, \Lambda)$, for each $p$, so that $\partial_{p-1} \circ \partial_{p}=0$ and such that the resulting differential complex $\left\{C_{p}(K, \Lambda), \partial_{p}\right\}_{p=0}^{r}$ calculates the homology of $|K|$. That is, if we define $H_{p}(C, \partial)=\operatorname{Ker}\left(\partial_{p}\right) \mid$ $\partial_{p+1}(C)$. In other words, $H_{p}(C, \partial) \cong H_{p}(|K|, \Lambda)$.

\section{Algebraic Discrete Morse Theory}

The aim of Discrete Morse Theory is to find simplicial collapses that transform a complex $K$ to a simpler one. This can be done using an essentially algebraic framework in which discrete Morse functions are convenient tools to keep track of the collapses, and the order in which they are done.

Now, we recover all the algebraic machinery underlying in Discrete Morse Theory, establishing a new framework for dealing with special chain complexes associated to finite cell complexes and we show that trees are a convenient combinatorial tool for solving the homological computation problem.

Definition 1. An integral chain complex $(C, d, \phi)$ is a graded module $C=\left\{C_{p}\right\}_{p=0}^{n}$ endowed with two linear maps: a differential operator $d: C_{*} \rightarrow C_{*-1}$, and an integral operator $\phi: C_{*} \rightarrow C_{*+1}$, satisfying the global nilpotency properties $d \circ d=0$ and $\phi \circ \phi=0$.

This integral operator, can also be called chain homotopy operator (Eilenberg and Mac Lane, 1953,1954). We will represent an integral operator by an arrow from the cell of lower dimension to the cell of higher dimension (see Fig. 2).

Definition 2. An integral chain complex $(C, d, \phi)$ is $d$-pure if the condition $d=d \circ \phi \circ d$ (called homology condition) is satisfied. An integral chain complex $(C, d, \phi)$ is $\phi$-pure if the condition $\phi=\phi$ $\circ d \circ \phi$ (called Strong Deformation Retract condition) is satisfied. An integral chain complex that is both, $d$-pure and $\phi$-pure, is called homology integral chain complex. In this case, $d$ (resp. $\phi$ ) is a homology differential (resp. integral) operator.

For instance, the integral chain complex in Fig. 3, is a homology integral chain complex (the conditions $d=d \circ \phi \circ d$ and $\phi=\phi$ $\circ d \circ \phi$ are satisfied for every cell of the complex).

Given two integral chain complexes $(C, d, \phi)$ and $\left(C^{\prime}, d^{\prime}, \phi^{\prime}\right)$, a map of integral chain complexes $\left.(f, g):(C, d, \phi) \Rightarrow C^{\prime}, d^{\prime}, \phi^{\prime}\right)$ is a couple of

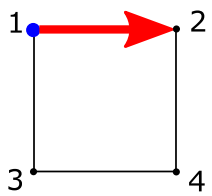

\begin{tabular}{c|c|c|}
$\sigma$ & $d$ & $\phi$ \\
\hline$\langle 1\rangle$ & 0 & $\langle 1,2\rangle$ \\
$\langle 2\rangle$ & 0 & 0 \\
$\langle 3\rangle$ & 0 & 0 \\
$\langle 4\rangle$ & 0 & 0 \\
$\langle 1,2\rangle$ & $\langle 1\rangle+\langle 2\rangle$ & 0 \\
$\langle 1,3\rangle$ & $\langle 1\rangle+\langle 3\rangle$ & 0 \\
$\langle 2,4\rangle$ & $\langle 2\rangle+\langle 4\rangle$ & 0 \\
$\langle 3,4\rangle$ & $\langle 3\rangle+\langle 4\rangle$ & 0
\end{tabular}

Fig. 2. An integral chain complex and an integral operator (represented by an arrow). 


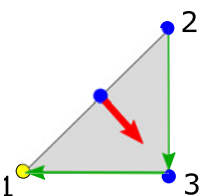

\begin{tabular}{c|c|c}
$\sigma$ & $d$ & $\phi$ \\
\hline$\langle 1\rangle$ & 0 & 0 \\
$\langle 2\rangle$ & 0 & $\langle 1,2\rangle+\langle 1,3\rangle$ \\
$\langle 3\rangle$ & 0 & $\langle 1,2\rangle$ \\
$\langle 1,2\rangle$ & $\langle 1\rangle+\langle 2\rangle$ & $\langle 1,2,3\rangle$ \\
$\langle 1,3\rangle$ & $\langle 1\rangle+\langle 3\rangle$ & 0 \\
$\langle 2,3\rangle$ & $\langle 2\rangle+\langle 3\rangle$ & 0 \\
$\langle 1,2,3\rangle$ & $\langle 1,2\rangle+\langle 1,3\rangle+\langle 2,3\rangle$ & 0
\end{tabular}

Fig. 3. A homology integral chain complex. The homology integral operator is represented by arrows.

linear maps $f: C \rightarrow C^{\prime}$ and $g: C^{\prime} \rightarrow C$ such that $f \circ d=d^{\prime} \circ f$, $g \circ d^{\prime}=d \circ g, f \circ \phi=\phi^{\prime} \circ f$ and $g \circ \phi^{\prime}=\phi \circ g$.

Definition 3. Given two integral chain complexes $(C, d, \phi)$ and $\left(C^{\prime}, d^{\prime}, \phi^{\prime}\right)$, we say that they are integral chain equivalent if there exists a map of integral chain complexes $(f, g)$, such that $f \circ g=i d_{C^{\prime}}-d^{\prime} \circ \phi^{\prime}-\phi^{\prime} \circ d^{\prime}$ and $g \circ f=i d_{C}-d \circ \phi-\phi \circ d$.

Two integral chain equivalent complexes are shown in Fig. 4.

The homology $H_{*}(C, d, \phi)$ of an integral chain complex $(C, d, \phi)$ is the graded abelian group $H_{*}(C)$, such that $\left(H_{*}(C), 0,0\right)$ is integral chain equivalent to $(C, d, \phi)$. The differential (resp. integral) homology of an integral chain complex $(C, d, \phi)$ is the homology of $(C, d, 0)$ (resp. the homology of $(C, 0, \phi))$. If $(C, d, \phi)$ is a homology integralchain complex, then $H_{*}(C, d, \phi) \sim H_{*}(C, d, 0) \sim H_{*}(C, 0, \phi)$.

The notion of pure integral chain complex is underlying in the work of Sergeraert $(1994)$, Forman $(1995,1998)$ and that of theory of discrete differential forms (Desbrun et al., 2006). The integral chain equivalence relation can be seen as the natural extension of the classical chain homotopy equivalence between chain complexes to the integral case (see, for example, (Eilenberg and Mac Lane, 1953,1954)).

The computation of the homology of a chain complex $(C, d)$ can be directly obtained from an integral operator $\phi: C_{*} \rightarrow C_{*+1}$, satisfying the strong deformation retract (SDR, for short) and homology conditions with regards to the differential operator $d$ (Gugenheim et al., 1989, 1991).

Proposition 1. Let $(C, d, \phi)$ be an integral chain complex. Let $\pi$ : $C_{*} \rightarrow C_{*}$ be the linear map (called the flow of $(C, d, \phi)$ ) defined by $\pi=i d_{C}-d \circ \phi-\phi \circ d$ and let $\Delta: C_{*} \rightarrow C_{*}$ be the linear map (called Laplacian of $(C, d, \phi))$ defined by $\Delta=d \circ \phi+\phi \circ d$. Then, the following properties hold:

(a) $d \circ \pi=d-d \circ \phi \circ d=\pi \circ d$ and $\phi \circ \pi=\phi-\phi \circ d \circ \phi=\pi \circ \phi$. In the case of a homology integral chain complex, $d \circ \pi=0=\pi \circ d$ and $\phi \circ \pi=0=\pi \circ \phi$.

(b) $d \circ \Delta=d \circ \phi \circ d=\Delta \circ d$ and $\phi \circ \Delta=\phi \circ d \circ \phi=\Delta \circ \phi$. In the case of a homology integral chain complex, $d \circ \Delta=d=\Delta \circ d$ and $\phi \circ \Delta=\phi=\Delta \circ \phi$.

(c) Given a p-chain $a$, we have the following equality $a=\pi(a)+$ $\Delta(a)$.

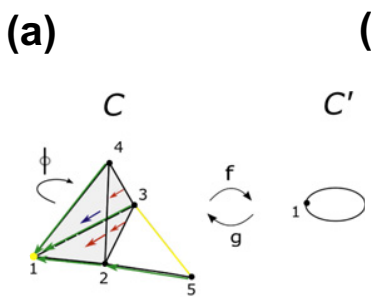

(b) \begin{tabular}{c|c|c}
$\sigma$ & $d(\sigma)$ & $\phi(\sigma)$ \\
\hline$\langle 2\rangle$ & $\langle 1,2\rangle$ & \\
\hline$\langle 3\rangle$ & $\langle 1,3\rangle$ & \\
\hline$\langle 4\rangle$ & $\langle 1,4\rangle$ & \\
\hline$\langle 5\rangle$ & $\langle 1,2\rangle+\langle 2,5\rangle$ & \\
\hline$\langle 2,3\rangle$ & $\langle 1,2,3\rangle$ & \\
\hline$\langle 2,4\rangle$ & $\langle 1,2,4\rangle$ & \\
\hline$\langle 3,4\rangle$ & $\langle 1,3,4\rangle$ & \\
\hline$\langle 2,3,4\rangle$ & $\langle 2,3\rangle+\langle 2,4\rangle$ & $\langle 1,2,3,4\rangle$ \\
& $+\langle 3,4\rangle$ & \\
\hline
\end{tabular}

Fig. 4. (a) Two integral chain equivalent complexes $(C, d, \phi)$ and $\left(C^{\prime}, d^{\prime}, \phi^{\prime}\right)$. (b) The $d$ and $\phi$ values of the complex $(C, d, \phi)$.
(d) $\left.\pi^{2}=\pi-\phi \circ(d-d \circ \phi \circ d)-d-d \circ \quad \phi \circ d\right) \circ \phi=\pi-d \circ(\phi$ $-\phi \circ d \circ \phi)-\phi-\phi \circ d \circ \phi) \circ d$.
(e) $\left.\Delta^{2}=d+\phi\right) \Delta(d+\phi)$.
(f) $\pi \circ \Delta=d-d \circ \phi \circ d) \circ \phi+\phi \quad \circ(d-d \circ \phi \circ d)=d \circ(\phi-\phi$ $\circ d \circ \phi)+\phi-\phi \circ d \circ \phi) \circ d=\Delta \circ \pi$.

Definition 4. The integral chain complex $\pi(C, d, \phi)=(\pi(C)$, $\left.\left.d\right|_{\pi(C)},\left.\phi\right|_{\pi(C)}\right)$ is the harmonic complex associated to $(C, d, \phi)$. If $(C, d, \phi)$ is a $d$-pure or a $\phi$-pure integral chain complex, then $\pi^{2}=\pi \circ \pi=\pi$ and $\pi(C)=\{x \in C \mid x=\pi(x)\}$.

In other words, the harmonic complex $\left(\pi(C),\left.d\right|_{\pi(C)}, 0\right)$ associated to a pure integral chain complex $(C, d, \phi)$ is formed by the $\pi$-equivariant chains of $C$. If $(C, d, \phi)$ is a homology integral chain complex, its harmonic complex is of the kind $(\pi(C), 0,0)$ and given any $p$ chain the chain map $\pi$ describes a representative cycle of the homology class associated to this $p$-chain.

In Fig. 4 two integral chain equivalent complexes $(C, d, \phi)$ and $\left(C^{\prime}, d^{\prime}, \phi^{\prime}\right)$ are shown. The complex $(C, d, \phi)$ on the left is a $\phi$-pure integral chain complex. The complex $\left(C^{\prime}, d^{\prime}, \phi^{\prime}\right)$ on the right is the harmonic complex of the complex $(C, d, \phi) .\left(C^{\prime}, d^{\prime}, \phi^{\prime}\right)$ is a homology integral chain complex $\left(d^{\prime}(\sigma)=0, \phi^{\prime}(\sigma)=0 \forall \sigma \in C^{\prime}\right)$.

Definition 5. The integral chain complex $\Delta(C, d, \phi)=\Delta(C)\left(\left.d\right|_{\Delta(C),} \phi\right.$ $\left.\left.\right|_{\Delta(C)}\right)$ is the Laplacian complex associated to $(C, d, \phi)$. If $(C, d, \phi)$ is a $d$-pure or $\phi$-pure integral chain complex, then $\Delta \circ \Delta=\Delta$ and $\Delta(C)=\{x \in C \mid x=\Delta(x)\}$.

In other words, the Laplacian complex $\Delta(C, d, \phi)$ associated to a pure integral chain complex $(C, d, \phi)$ is formed by all the $\Delta$-equivariant chains.

Proposition 2. If $(C, d, \phi)$ is a (differential or integral) pure integralchain complex, we can derive the following properties:

(p1) $\pi \circ \Delta=0=\Delta \circ \pi$.

(p2) $(C, d, \phi)=\pi(C, d, \phi) \oplus \Delta(C, d, \phi)$ as integral-chain complexes. In particular, $\operatorname{Ker} \Delta=\pi(C)$ and $\Delta(C)=\operatorname{Ker} \pi$.

(p3) $\Delta(C)=\phi(C) \oplus d \circ \phi)(C)$ as graded modules.

In order to emphasize the dependency of $\pi$ and $\Delta$ with regards to $d$ and $\phi$, we will denote these maps by $\pi_{(d, \phi)}$ and $\Delta_{(d, \phi)}$, respectively.

The following proposition will be fundamental in developing an integral-chain framework for Discrete Morse Theory. In fact, it shows that to use pure integral operators as chain homotopies decomposing finitely generated chain complexes is a key point:

Proposition 3. If $(C, d, \phi)$ is a (differential or integral) pure integralchain complex, we have that

$\operatorname{Ker} \phi \cong \pi(C) \oplus \phi(C) \cong \operatorname{Ker} \Delta(C) \oplus \phi(C)$

as graded modules. that

In particular, a map of integral-chain complexes $(f, g)$ satisfies $f \circ \pi_{(d, \phi)}=\pi_{\left(d^{\prime}, \phi^{\prime}\right)} \circ f, g \circ \pi_{\left(d^{\prime}, \phi^{\prime}\right)}=\pi_{(d, \phi)} \circ g, f \circ \Delta_{(d, \phi)}=\Delta_{\left(d^{\prime}, \phi^{\prime}\right)} \circ f$ and $g \circ \Delta_{\left(d^{\prime}, \phi^{\prime}\right)}=\Delta_{(d, \phi)} \circ g$. That is, $f$ and $g$ are compatible with regards to the respective flows and Laplacians.

In spite of its simplicity, the following result is essential for developing our homological theory of integral-chain complexes:

Lemma 1 (Integral-chain Lemma). An integral chain complex $(C, d, \phi)$ is integral-chain equivalent to its harmonic complex $\pi(C, d, \phi)$. This last harmonic complex $\pi(\mathcal{C}, d, \phi)$ is of the form $\left(\pi(C), d_{\pi}, \phi_{\pi}\right)$ where $d_{\pi}(\pi(x))=d-(d \circ \phi \circ d)(x)$ and $\phi_{\pi}(\pi(x))=\phi$ $-(\phi \circ d \circ \phi)(x)$. 
Proof. Let $f: C \rightarrow \pi(C)$ be the linear map defined by $f(x)=\pi(x), \forall x \in C_{*}$. Let $g: \pi(C) \rightarrow C$ be the linear map defined by $g(x)=x, \forall x \in \pi(C)$. Then, it is a simple exercise to show that $(f, g)$ is a couple of maps of integral chain complexes which induces the integral-chain equivalence. The rest of assertions can be directly deduced from Proposition 1 (a).

Corollary 1. The harmonic complex $\pi(C, d, \phi)$ associated to a d-pure (resp. $\phi$-pure) integral-chain complex $(C, d, \phi)$ is of the form $\left(\pi(C), 0, \phi_{\pi}\right)\left(\operatorname{resp} .\left(\pi(C), d_{\pi}, 0\right)\right), \phi_{\pi}(\pi(x))=\phi-(\phi \circ d \circ \phi)(x)$ (resp. $\left.d_{\pi}(\pi(x))=d-(d \circ \phi \circ d)(x)\right)$.

An example of Lemma 1 can be seen in Fig. 4 , where $(C, d, \phi)$ is a $\phi$-pure integral-chain complex, and $\left(C^{\prime}, d^{\prime}, \phi^{\prime}\right)$ is its harmonic complex.

Now, we give some definitions related to integral-chain perturbation of complexes.

Definition 6. An integral chain complex $(C, d, \phi)$ is called $d$ pointwise nilpotent (resp. $\phi$-pointwise nilpotent) if for any $a \in C$ there is some $n(a) \in \mathbb{N}$ with $d \circ\left(i d_{C}-d \circ \phi-\phi \circ d\right)^{n(a)}=0$ (resp. with $\left.\phi \circ\left(i d_{C}-d \circ \phi-\phi \circ d\right)^{n(a)}=0\right)$. The smallest value for $n(a)$ is called the degree of differential (resp. integral) nilpotency of $a$.

Proposition 4. Given an $\phi$-pointwise (resp. d-pointwise) nilpotent chain complex $(C, d, \phi)$, it is integral chain equivalent to a $\phi$-pure (resp. $d$-pure) integral chain complex $(C, d, \tilde{\phi})(\operatorname{resp} .(C, \tilde{d}, \phi))$.

Proof. We only prove the existence of the $\phi$-pure integral chain complex $(C, d, \tilde{\phi})$. The other result can be derived directly from the fact that $(C, \phi, d)$ is also a $\phi$-pointwise nilpotent integral-chain complex. Define $\tilde{\phi}: C_{*} \rightarrow C_{*+1}$ by $\tilde{\phi}=\sum_{k \geqslant 0} \phi \circ\left(i d_{C}-d \circ \phi\right)^{k}$. This is well defined due to the pointwise nilpotency of $(C, d, \phi)$, since all but finitely many terms vanish on the right hand side. The map $\tilde{\phi}$ satisfies $\tilde{\phi} \circ \tilde{\phi}=0$ and the SDR condition $\tilde{\phi} \circ d \circ \tilde{\phi}=\tilde{\phi}$. The couple of maps $\left(\pi(d, \tilde{\phi}), i d_{c}\right)$ establishes the integral chain equivalence between $(C, d, \phi)$ and $(C, d, \tilde{\phi})$.

From now on, all the integral chain complexes considered in the paper will be $\phi$-pointwise nilpotents. Analogous results can be determined for $d$-pointwise nilpotent chain complexes.

In the next section, we define some notions in order to do the link with the algebraic work underlying in Discrete Morse Theory.

\section{Discrete Morse Theory and optimality}

In this section, we will show that all the results above can be used as a tool for reaching interesting combinatorial results in DMT.

Definition 7. Let $(K, \partial)$ a finite cell complex. An operator $h$ : $C_{*}(K) \rightarrow C_{* \pm r}(K)$ is said to be combinatorial if $\forall p-$ cell $\sigma^{(p)}$, $h\left(\sigma^{(p)}\right)=\lambda \beta^{(p \pm r)}$, where $\lambda \in \mathbb{Z}$ and $\beta$ is a $(p \pm r)-$ cell.

Now, we give some basic notions of DMT with some slight modifications and without using, in principle, discrete Morse functions.

Definition 8. A combinatorial vector field $\mathcal{V}$ defined on a connected cell complex $K$ is a collection of disjoint pairs of cells $\left\{\alpha^{(p)}<\beta^{(p+1)}\right\}$.

Definition 9. A $\mathcal{V}$-path or gradient path $\gamma$ is an alternating sequence of cells $a_{0}^{(p)}, b_{0}^{(p \pm 1)}, a_{1}^{(p)}, b_{1}^{(p \pm 1)}, a_{2}^{(p)}, \ldots$, such that for each pair of consecutive cells, one is a facet of the other, and the following condition is satisfied: either $\left\{a_{i}^{(p)}<b_{i}^{(p \pm 1)}\right\}$ or $\left\{b_{i}^{(p \pm 1)}<a_{i+1}^{(p)}\right\}$ belongs to $\mathcal{V}, \forall i \geqslant 0$.
If the final cell in the gradient path $\gamma$ above is $\alpha_{r}^{(p)}$, then we say that $\gamma$ has length $r$ Forman (2002). If it ends at $\beta_{r}^{(p \pm 1)}$ then we say that $\gamma$ has length $r \frac{1}{2}$. If the cells $b_{i}$ of the gradient path $\gamma$ are of dimension $p+1$ and it has length $r \frac{1}{2}$, the gradient path $\gamma$ is called upper $\mathcal{V}$-path or upper gradient path. For any cells $a$ and $b$, let $\Gamma(a, b)$ denote the set of gradient paths from $a$ to $b$ (of any length), i.e., such that the first cell in the sequence is $a$ and the last cell in the sequence is $b$. A $\mathcal{V}$-path is non trivial and closed if $r \geqslant 1$ and the first and last cells in the sequence are the same.

Definition 10. A discrete gradient vector field is a combinatorial vector field with non trivial closed $\mathcal{V}$-paths. In this way, it can be seen as an acyclic cells pairing. A cell $\alpha$ is a critical cell of $\mathcal{V}$ if it is not paired with any other cell in $\mathcal{V}$.

Definition 11. A combinatorial integral operator defined on a cell complex $K$ is a collection of disjoint pairs of (not necessary incident) cells $\left\{\alpha^{(p)}, \beta^{(p+1)}\right\}$ of the same connected component.

Therefore, a discrete gradient vector field is a special kind of combinatorial integral operator.

Forman, 1995, 1998 proved that the topology of a discrete manifold is related to the critical cells of a discrete function defined on it, mimicking the results of Morse in the smooth case. The number of critical cell depends on the discrete gradient vector field considered (see Fig. 5). In (Lewiner et al., 2003), the problem of the optimality (that is minimizing the number of critical cells for combinatorial vector fields) on a 2-manifold is analyzed using Hasse diagram and hypergraph tools. However, this problem has not been solved for the general case.

If we restrict ourselves to use DMT techniques, it is not always possible to obtain a number of critical cells that coincides with the Betti numbers of the complex. This is the case of the Bing's house and the Dunce hat complexes, that are contractible but not collapsible (see (Ayala et al., 2010)).

The next results will show that by using integral operators for chain complexes, we can solve this problem, and always reduce the initial complex to the minimum number of critical cells, that corresponds with the Betti numbers. This means that we are able to guarantee homological optimality (what is called perfection in the DMT context, see Ayala et al. (2010)).

Definition 12. Given an upper gradient path $\gamma=\Gamma(a, b)$ formed by the alternating sequence $\gamma: a_{0}^{(p)}, b_{0}^{(p+1)}, a_{1}^{(p)}, b_{1}^{(p+1)}, \ldots, a_{t}^{(p)}, b_{t}^{(p+1)}$ its corresponding chain homotopy path is defined by the sequence $b_{0}^{(p+1)}, b_{1}^{(p+1)}, \ldots, b_{t}^{(p+1)}$.

First, the combinatorial integral operators derived from combinatorial vector fields are $\phi$-pointwise nilpotent.

Proposition 5. A discrete gradient vector field $\mathcal{V}$ gives rise to a $\phi$-pointwise nilpotent integral-chain complex $(C(K), d, V)$.

Proof. Let us emphasize that two pairs $\{a, b\}$ and $\left\{a^{\prime}, b^{\prime}\right\}$ of $\mathcal{V}$ have no elements in common. The combinatorial vector field $\mathcal{V}$ gives rise to a linear map $V: C_{*}(K) \rightarrow C_{*+1}(K)$, defined by $V(a)=b$ if $\{a, b\} \in \mathcal{V}$ and $V(a)=0$ for the rest of the cells. It is clear that $V \circ V=0$. The map $V$ is an integral operator for $C_{*}(K)$. It is straightforward to prove that $(C(K), d, V)$ is $\phi$-pointwise integral-chain complex.

Combining Propositions 1-3, we assert the following result which is the key for reinterpreting DMT in terms of an integralchain complex: 

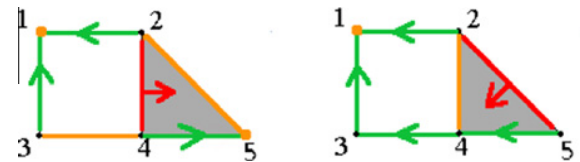

Fig. 5. A cell pairing on the left $(\langle 1\rangle,\langle 5\rangle,\langle 3,4\rangle$ and $\langle 2,5\rangle$ are critical), and an optimal one on the right $(\langle 1\rangle$ and $\langle 2,4\rangle$ are critical). The pairing is represented with an arrow from the cell of lower dimension to its paired cell of higher dimension.
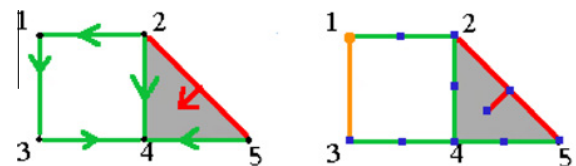

Fig. 6. A combinatorial vector field (on the left). On the right a gradient set of trees where cells $\langle 1\rangle$ and $\langle 1,3\rangle$ do not belong to the forest, $\langle 2,5\rangle$ and $\langle 2,4,5\rangle$ belong to the tree of dimension $1-2$ and the rest of cells belong to the tree of dimension $0-1$.

Proposition 6. If $(C, d, V)$ is a $\phi$-pointwise nilpotent integral-chain complex being $V$ a gradient vector field, then there is an integral-chain equivalent $\phi$-pure complex $(C, d, \widetilde{V})$, such that its harmonic complex $\pi(C, d, \widetilde{V})=\left(\operatorname{Ker} \widetilde{V} \backslash \widetilde{V}(C), d_{\pi}, 0\right)$.

This last integral-chain complex is constituted by finite linear combinations of the different critical cells of $V$ and $d_{\pi}$ can be seen as the boundary operator of the corresponding cell complex determined by the critical cells, also called harmonic Morse cell complex $\mathcal{M}(C, d, V)$ associated to $(C, d, V)$. Analogously, the Laplacian complex $\Delta(C, d, \widetilde{V})$ can be seen as the acyclic chain complex of the cell complex $\mathcal{M}(C, d, V)$, also called Laplacian Morse complex associated to $(C, d, V)$. Moreover, its boundary operator $\partial_{\mathcal{M}}$ is determined by $\partial_{\mathcal{M}}\left(\Delta\left(\sigma^{(p)}\right)\right)=d \circ \widetilde{V} \circ d\left(\sigma^{(p)}\right), \forall \sigma^{(p)} \in C$.

Proof. Due to Propositions 3 and 1 and defining $\widetilde{V}: C_{*} \rightarrow C_{*+1}$ by $\widetilde{V}=\sum_{k=0}^{t} V \circ\left(i d_{C}-d \circ V\right)^{k}=\sum_{k=0}^{t}\left(i d_{C}-V \circ d\right)^{k} V$, we have that

\section{$(f, g): \operatorname{Ker} \widetilde{V} \backslash \widetilde{V}(C) \cong \pi(C, d, \widetilde{V})$}

is an isomorphism of chain complexes, with $f: \operatorname{Ker} \widetilde{V} \backslash \widetilde{V}(C) \rightarrow$ $\pi(\mathcal{C}, d, \phi)$ and $g: \pi(C, d, \widetilde{V}) \rightarrow \operatorname{Ker} \widetilde{V} \backslash \widetilde{V}(C)$ respectively defined by $f(\sigma)=\pi_{\widetilde{V}(d, \widetilde{V})}(\sigma)=\sigma-\widetilde{V} \circ d(\sigma), \forall \sigma \in: \operatorname{Ker} \widetilde{V} \backslash \widetilde{V}(C)$ and $g\left(\pi_{(d, \widetilde{V})}\right)(\beta)=$ $\beta-d \circ \widetilde{V}(\beta), \forall \beta \in C$.

Now, let us prove that $\operatorname{Ker} \widetilde{V}=\operatorname{Ker} V$ and $\widetilde{V}(C)=V(C)$.

It is clear that $\operatorname{Ker} V \subset \operatorname{Ker} \widetilde{V}$. Let $x \in \operatorname{Ker} \widetilde{V}$ be an element such that $x \notin \operatorname{Ker} V$. That means that $\sum_{k=0}^{t} \pi(d, V)^{k}(x) \in \operatorname{Ker} V$. That implies that $V\left(i d_{C}-\pi^{t+1}\right)(x)=V(d \circ V+V \circ d) \circ\left(\sum_{k=0}^{t} \pi(d, V)^{k}\right)$ $(x)=0$ and, then $V(x)=V \pi^{t+1}(x)=0$. In a similar manner, it is possible to deduce that $\widetilde{V}(C)=V(C)$ and that it also admits a combinatorial basis.

Let us now prove that the chain complex $(\widetilde{V}(C) \oplus(d \circ \widetilde{V})(C), d)$ is acyclic. We have that $d(\widetilde{V}(C)) \subset(d \circ \widetilde{V})(C)$ and $d((d \circ \widetilde{V})(C))=0$. Now, let us suppose that there is a chain $x=x^{\prime}+x^{\prime} \in \widetilde{V}(C) \oplus(d \circ \widetilde{V})(C)$ such that $d(x)=0$. This means that $d\left(x^{\prime}\right)=0$. Since $x^{\prime}=\widetilde{V}(z)$, then $(d \circ \widetilde{V})(z)=0$. Due to the fact that the integral operator satisfy the SDR condition $\widetilde{V} \circ d \circ \widetilde{V}=\widetilde{V}$, we conclude that $x^{\prime}=0$.

Finally, the boundary operator of $\mathcal{M}(C, d, V)$ is the differential operator $d$ restricted to the complex and its acyclicity can be proved using Proposition 3.

Let us note that $H_{*}(\mathcal{M}(C, d, V)) \cong H_{*}(K, \Lambda)$. Moreover, the boundary operator $d_{\pi}$ of the Morse cell complex $\mathcal{M}(C, d, V)$ has a clear interpretation in terms of gradient paths of $\widetilde{V}$.

Proposition 7. In the conditions of Proposition 6, and given a p-cell $\alpha, \widetilde{V}(\alpha)$ is a chain homotopy path.

In (Molina-Abril and Real, 2011), an integral operator $\phi$ giving rise to a homology integral chain complex is determined from a filtered cell complex by using an incremental technique. Given a $p$-cell $\sigma, \phi(\sigma)$ is a sum of (p+1)-cells in which at least one cell $\tau$ satisfies that $\sigma \in \partial(\tau)$. This operator $\phi$ gives rise in a natural way to a combinatorial integral operator on $K$.

Due to the fact that $\widetilde{V}(C)$ admits a combinatorial basis, and the chain complex $\widetilde{V}(C) \oplus(d \circ \widetilde{V}(C), d)$ is acyclic, we can assume that the sum $\omega$ of the elements in the combinatorial basis of $\widetilde{V}(C)$ satisfies that $d(\omega)=0$. That means that $\omega$ can be represented in terms of graphs using trees. In these trees, the nodes are $p$-cells and $(p+1)$-cells $\forall p \geqslant 0$ of the complex. The neighbors of a $p$-cell are $(p+1)$-cells and vice versa (see Fig. 6). This forest, is a representation in homological terms of the cell complex $K$, and it is called homological spanning forest or HSF, for short (see (Molina-Abril and Real, 2009, 2011)).

Given a HSF, it is possible to distinguish two kind of trees: homologically essential and inessential trees. In a homologically inessential tree the number of $p$-cells is the same as the number of $p+1$-cells. In a homologically $c$-essential tree, the difference between the number of $p$-cells and $p+1$-cells is a positive integer $c$. In this last case there exist $c p$-cells within this tree that represent a critical cell, that is, a homology generator. Therefore, given a HSF, a combinatorial integral operator can be directly deduced by maximally pairing each $p$-cell with a $(p+1)$-cell using some specific (a)

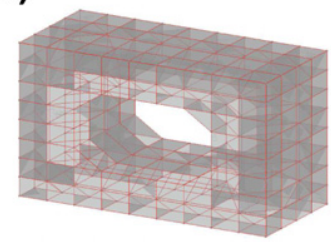

(b)

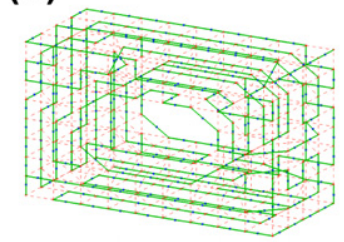

(c)

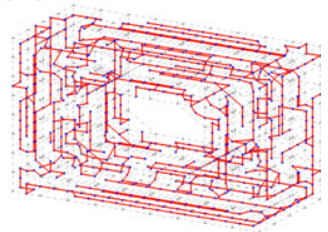

(d)

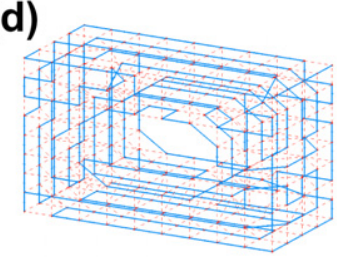

(e)

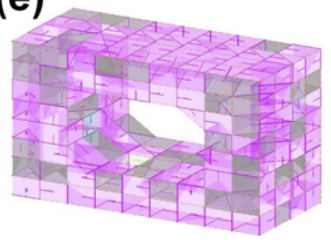

Fig. 7. A torus cell complex, part of its homological spanning forest representation, and the obtained optimal combinatorial pairing. 
(a)
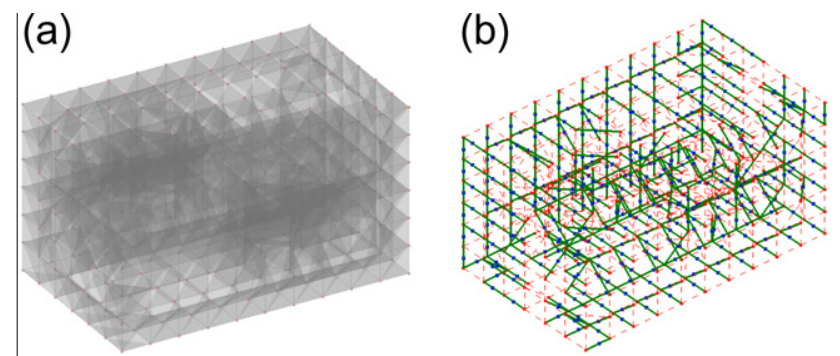

(c)

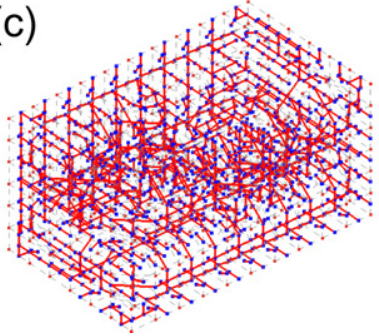

(d)

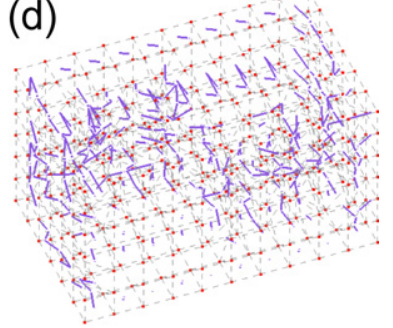

Fig. 8. A bing's house cell complex (a) and its homological spanning forest representation (b) $0-1$, (c) 1-2 and (d) 2-3 cells).

strategy (eventually, allowing the pairing of non-incident cells) for each homologically essential or inessential tree. In this process, only $c p$-cells (critical cells) of a homologically c-essential tree will remain unpaired.

Let us emphasize that the notion of optimality here is guaranteed in terms of finding a combinatorial integral operator. Therefore, the minimum number of critical cells will always coincide with the Betti numbers. In the pairing process, we might find some pairs of non-incident cells $\{\alpha, \beta\}$. In order to obtain optimality in the sense of Forman (pairing of incident cells), classical cancellation results (see (Forman, 1998)) involving the single path joining $\alpha$ and $\beta$ can be applied.

\section{Examples}

In this section, some examples showing the previous results are presented. We have developed a $\mathrm{C}++$ implementation that allows us to visualize and better understand them.

In Fig. 7 a) we can see the cell complex $K$ of a torus. Fig. 7 b) and c) represent the tree structure of a chain homotopy operator $\phi$ described over the $\{0$-cells, 1 -cells $\}$ and the $\{1$-cells, 2 -cells $\}$ of the initial complex respectively. The corresponding optimal combinatorial pairing, is shown in Fig. 7 d) and e). The different colors in Fig. 8 e) represent the different trees of the homological spanning forest of 1 -cells and 2-cells. The triple $(C(K), \partial, \phi)$ is a homology integral-chain complex.

In Fig. 8 the results obtained for an example of the bing's house with two rooms is shown. Due to the fact that this example is a contractible complex which is not collapsible, it is not possible to get an optimal discrete gradient vector field. Nevertheless, we obtain a combinatorial integral operator involving all the cells from its associated HSF structure, guaranteeing homological optimality.

\section{Conclusions}

In this paper, a graph version of the notion of optimal discrete gradient vector field is appropriately integrated into a new frame-

work of computational homological algebra that is suitable for advanced (algebraic) topological computation. A homology-based heuristic approach to find optimal gradient vector fields is also carried out. Some relations between classical algebraic topology, discrete differential form techniques Desbrun et al. (2006) and Discrete Morse Theory have been established, and a common dictionary of terms has been defined. Geometric (local and global curvature, geodesics, Ricci flow,...) and analytical information (area, volume, ...) of a finite cell complex $K$ embedded in $\mathbb{R}^{n}$ can be derived from an optimal discrete gradient vector field or, equivalently, its homological spanning forest, if we fix coordinates to each of its nodes (cells) and we install a metric on its space of chains. In future work, we plan to exploit the interplay of this framework with geometric or analytical information and to introduce new algebraic operators in our homological algebra framework "measuring" advanced algebraic-topological invariants.

Finally, the mathematical approach we develop here opens a door for advancing in the topological recognition of discrete structures and images, defining models in which combinatorics, algebra, analysis, geometry and topology ingredients are armonious and efficiently combined.

\section{Acknowledgements}

This work has been partially supported by the Andalusian Research Group CATAM FQM-296, the Spanish MICINN Research Project MTM2009-12716 and the Austrian Science Fund under grant P20134-N13.

\section{References}

Ayala, R., Fernandez-Ternero, D., Vilches, J., 2010. Perfect discrete morse functions on 2-complexes. Imagen-a 1, 19-25.

Desbrun, M., Kanso, E., Tong, Y., 2006. Discrete differential forms for computational modeling. In: SIGGRAPH '06: ACM SIGGRAPH 2006 Courses. ACM, New York, NY, USA, pp. 39-54.

Eilenberg, S., Mac Lane, S., 954. On the groups $\mathrm{H}(\pi, n)$, i, ii, iii. Ann. Math. 58, 55-106, $60,48-139,60,513-557$.

Forman, R., 1995. A Discrete Morse Theory for cell complexes. In: Yau, S.T. (Ed.), Topology and Physics for Raoul Bott. International Press.

Forman, R., 1998. Morse theory for cell complexes. Adv. Math. 134, 90-145.

Forman, R., 2002. Discrete Morse Theory and the cohomology ring. Trans. Am. Math. Soc. $354,5063-5085$.

González-D́raz, R., Jiménez, M.J., Medrano, B., Real, P., 2009. Chain homotopies for object topological representations. Discrete Appl. Math. 157 (3), 490-499.

González-Díraz, R., Real, P., 2005. On the cohomology of 3D digital images. Discrete Appl. Math. 147 (2-3), 245-263.

Gugenheim, V.K.A.M., Lambe, L.A., Stasheff, J.D., 1989. Perturbation theory in differential homological algebra. Illinois J. Math. 33, 357-373.

Gugenheim, V.K.A.M., Lambe, L.A., Stasheff, J.D., 1991. Perturbation theory in differential homological algebra. ii. Illinois J. Math. 35 (3), 357-373.

Hatcher, A., 2001. Algebraic Topology. Cambridge University Press, Canbridge, UK.

Lewiner, T., Lopes, H., Tavares, G., Matmídia, L., 2003. Towards optimality in Discrete Morse Theory. Exp. Math. 12, 2003.

Milnor, J., 1963. Morse theory. Ann. Math., 51.

Molina-Abril, H., Real, P., 2009. Homological computation using spanning trees. In: Bayro-Corrochano, E., Eklundh. J.-O. (Eds.), Progress in Pattern Recognition, Image Analysis, Computer Vision, and Applications. Vol. 5856 Lecture Notes in Computer Science. Springer, Berlin/ Heidelberg, pp. 272-278.

Molina-Abril, H., Real, P., 2011. A homological-based description of subdivided $n$ D objects. In: Real, P., Diaz-Pernil, D., Molina-Abril, H., Berciano, A., Kropatsch, W. (Eds.), Computer Analysis of Images and Patterns. Vol. 6854 Lecture Notes in Computer Science. Springer, Berlin/ Heidelberg, pp. 42-50.

Sergeraert, F., 1994. The computability problem in algebraic topology. Adv. Math. 104, 1-29. 